\title{
Initial manifestations and short term follow-up results of Henoch-Schönlein purpura in children: A report from two centers
}

\author{
(D) Kubra Ozturk, ${ }^{1}$ (i) Mustafa Cakan ${ }^{2}$ \\ ${ }^{1}$ Department of Pediatric Rheumatology, Gaziantep Cengiz Gokcek Children Hospital, Gaziantep, Turkey \\ ${ }^{2}$ Department of Pediatric Rheumatology, Sanliurfa Training and Research Hospital, Sanlıurfa, Turkey
}

\begin{abstract}
OBJECTIVE: This retrospective observational study aims to demonstrate initial signs and symptoms of Henoch-Schönlein purpura (HSP), search for risk factors for gastrointestinal and renal involvement and give short term follow-up results.

METHODS: The files of newly diagnosed HSP patients from two pediatric rheumatology centers in the southeastern part of the country were retrospectively analyzed in this study. Demographic, clinical features and laboratory results were recorded from the files.

RESULTS: The cohort consisted of 323 children (males: $53.6 \%$, females: $46.4 \%$ ). Median age at the time of diagnosis was $7.5(1.8-17.3)$ years and the median duration of follow-up was six (3-22) months. The rash was present in all cases but was not the first symptom in $22.9 \%$ of the cases. Arthritis and abdominal pain before the development of rash were the initial symptoms in $11.8 \%$ and $11.1 \%$ of the cases, respectively. Other manifestations were subcutaneous edema (63.2\%), arthralgia (57.6\%), arthritis (27.6\%), myalgia (17.6\%), lethargy (10.2\%), orchitis (7.5\%) and fever (5.3\%). Gastrointestinal involvement was seen in $53.3 \%$ and renal involvement in $23.5 \%$ of the cases. None of the patients developed renal impairment during the follow-up. Older age at diagnosis, necrotic rash, subcutaneous edema, abdominal pain, lethargy, myalgia, arthralgia and arthritis were significantly higher in patients with renal involvement, but none of the demographic, clinical and laboratory features was an independent risk factor for renal or gastrointestinal involvement.
\end{abstract}

CONCLUSION: Abdominal pain, arthritis may be the first manifestation of HSP. Having constitutional symptoms, such as fever, myalgia and lethargy at the time of diagnosis, may be warning signs of a more aggressive course with gastrointestinal and renal involvement.

Keywords: child; Henoch-Schönlein purpura; IgA vasculitis.

Cite this article as: Ozturk K, Cakan M. Initial manifestations and short term follow-up results of Henoch-Schönlein purpura in children: A report from two centers. North Clin Istanb 2020;7(4):341-347.

Lenoch-Schönlein purpura (HSP), also known as 1 IgA vasculitis, is the most common form of systemic vasculitis in children [1]. HSP is grouped under the rubric of small vessel vasculitis and typically involves skin, joints, kidneys and gut. The clinical spectrum may range from mild skin rash and arthralgia to severe gastrointestinal (GI) and renal involvement [1,2]. All of the patients have a purpuric rash typically located on the legs and buttocks. The rash may also extend to the arms and face [1-3]. Sometimes, GI manifestations, such as abdominal pain or join manifestations like arthralgia or arthritis, may precede the development of a purpuric rash in less than $5 \%$ of the patients [3]. Other manifestations are low-grade fever, lethargy, myalgia, orchitis, sub-

Received: October 26, 2019 Accepted: December 31, 2019 Online: June 01, 2020

Correspondence: Mustafa CAKAN, MD. Sanliurfa Egitim ve Arastirma Hastanesi, Cocuk Romatoloji Klinigi, 63250 Eyyubiye, Sanliurfa, Turkey.

Tel: +90 4143171717 e-mail: mustafacakan@hotmail.com

(c) Copyright 2020 by Istanbul Provincial Directorate of Health - Available online at www.northclinist.com 
cutaneous edema, and rarely pulmonary hemorrhage and central nervous system involvement $[1,3]$.

The main cause of morbidity is renal involvement that is seen around $20-60 \%$ of the patients. Most of the cases with renal involvement have microscopic hematuria and/ or non-nephrotic proteinuria that resolve spontaneously [3]. Fewer than $5 \%$ of the children with renal involvement progress to end-stage renal failure [1]. Renal involvement is most commonly seen in the first few weeks of the disease, but monitoring up to two years is recommended by many experts [1-4].

This retrospective observational study aims to demonstrate initial signs and symptoms of HSP, search for risk factors for GI and renal involvement and give short term follow-up results from two pediatric rheumatology centers in the southeastern part of Turkey.

\section{MATERIALS AND METHODS}

The files of children diagnosed with HSP from two pediatric rheumatology centers between July 2017 and May 2019 were retrospectively reviewed. To be included in this study, the child had to be diagnosed as having HSP by either one of the pediatric rheumatologists, had to be completed initial three months after diagnosis, and had to be regularly coming to follow-up visits. All children were fulfilling the classification criteria of the EULAR/ PRINTO/PReS-endorsed Ankara 2008 criteria [5]. Gender, age at diagnosis, the month of the diagnosis, duration of follow-up, initial clinical features, initial laboratory tests, GI and renal involvement during the disease course were recorded from the files.

Gastrointestinal involvement was defined as colicky abdominal pain and/or GI bleeding. Renal involvement was defined as microscopic hematuria (urine red blood cells $>5 /$ high power field in a centrifuged specimen or $\geq 1(+)$ hemoglobin on dipstick testing), non-nephrotic proteinuria $(\geq 1(+)$ protein on dipstick testing, protein/creatinine ratio $\geq 0.2$ on early morning spot urine test, or $>4 \mathrm{mg} / \mathrm{m}^{2} /$ hour in 24-hour urine). Nephrotic syndrome was defined as proteinuria $>40 \mathrm{mg} / \mathrm{m}^{2} /$ hour accompanied by hypoalbuminemia and/or edema. Nephritic syndrome was defined as hematuria, impaired glomerular filtration rate, oliguria, hypertension and edema. This study was approved by the local ethics committee (date: 04.11.2019, number: 19/04/01) and was conducted according to the tenets of the declaration of Helsinki.

\section{Statistical Analysis}

The SPSS version 21.0 (SPSS, Inc., Chicago, IL, USA) was used for statistical analysis. The variables were investigated using visual (histogram, probability plots) and analytic methods (Kolmogorov-Smirnov/ShapiroWilk's test) to determine whether or not they were normally distributed. Quantitative data with non-normal distribution were presented as median and minimummaximum. Categorical data were presented as counts and percentages. Categorical variables were compared using the Chi-square test or Fisher's exact test where appropriate. The Mann-Whitney $U$ test was used to compare the non-normally distributed continuous data between two groups. Multiple linear logistic regression model was used to identify the independent predictor factors for gastrointestinal and renal involvement. A pvalue of less than 0.05 was considered to show a statistically significant result.

\section{RESULTS}

The cohort consisted of 323 children. There was slightly male dominance (males: $53.6 \%$, females: $46.4 \%$ ). Median age at the time of diagnosis was $7.5(1.8-17.3)$ years. Median duration of follow-up was 6 (3-22) months. Seasonal distribution showed that spring (31.2\%) and winter $(31.2 \%)$ were the most common months of the year in HSP diagnosis, followed by autumn $(26.1 \%)$ and summer $(11.4 \%)$. The history of recent upper respiratory tract infection was present in $25.7 \%$, and a history of acute gastroenteritis was present in $4.0 \%$ of the cases. The rash was the first symptom in $77.1 \%$ of the cases. Arthritis and abdominal pain before the development of rash were the initial symptoms in $11.8 \%$ and $11.1 \%$ of the cases, respectively. Clinical manifestations are shown in Table 1. All patients had typical HSP rash. The most common location of the rash was upper and lower legs (70.3\%) followed by rash extending to the arms (14.9\%), located only on the lower legs $(8.0 \%)$, extending to the face $(3.7 \%)$, and extending to the torso $(3.1 \%)$. Bullous rash was observed in $8.0 \%$ of the cases and necrotic rash, most commonly located around the ankles, was observed in $5.0 \%$ of the cases. Median duration of healing of the rash was 3.0 (1-16) weeks. In 11 patients, the rash healed in two months, and in only one case, it took four months.

Other initial manifestations were subcutaneous edema (63.2\%), arthralgia (57.6\%), arthritis $(27.6 \%)$, myalgia (17.6\%), lethargy (10.2\%), orchitis $(7.5 \%)$ and fever $(5.3 \%)$. GI involvement in the form of moderate to severe 
TABLE 1. Clinical manifestations of the children with HenochSchönlein purpura

\begin{tabular}{lc} 
Clinical manifestation & $\%$ \\
\hline Rash & 100 \\
Subcutaneous edema & 63.2 \\
Lethargy & 10.2 \\
Fever & 5.3 \\
Myalgia & 17.6 \\
Arthralgia & 30.0 \\
Arthritis & 27.6 \\
Vomiting & 18.9 \\
Abdominal pain & 53.3 \\
Gastrointestinal bleeding & 8.0 \\
Orchitis & 7.5 \\
Renal involvement & 23.5
\end{tabular}

abdominal pain that was affecting the daily activities or awakening the child from sleep was observed in $53.3 \%$ of the cases. GI bleeding in the form of hematochezia and/ or melena was observed in $8.0 \%$ of the cases. One patient developed intussusception and perforation and needed surgical repair. Also, one patient had convulsion secondary to normotensive posterior reversible encephalopathy syndrome that healed without any neurologic sequela.

Ninety-one patients (28.2\%) were hospitalized mostly due to abdominal pain or GI bleeding. Severe arthritis and/or myalgia that were affecting the ambulation of the patients were the cause of hospitalization in seven patients $(2.1 \%)$. Corticosteroids were used in $44.9 \%$ of the whole cohort. Abdominal pain $(66.7 \%)$ and GI bleeding (17.2\%) were the most common indications for corticosteroids and other indications were extensive skin involvement (7.5\%) and severe arthritis (6.2\%).

Any type of renal involvement at the beginning or during the disease course was observed in $23.5 \%$ of the cohort. Urine analysis was abnormal in $15.2 \%$ of the patients at the time of diagnosis. Type of abnormality was microscopic hematuria (57.2\%), microscopic hematuria and proteinuria (24.5\%) and isolated proteinuria (18.3\%). In $8.3 \%$ of the cases, either one of the above mentioned renal involvement developed during the follow-ups. Nephrotic range proteinuria was observed only in five cases $(1.5 \%)$ and the nephritic syndrome was not observed in any case. At the last visit, persistent renal involvement was seen in 30 patients $(9.3 \%)$ consisting of non-nephrotic proteinuria (46.6\%), microscopic hematuria (26.7\%),
TABLE2. Complete blood count and acute phase reactant results of the patients at the time of diagnosis

\begin{tabular}{lc} 
& Median (min.-max.) \\
\hline White blood cell count $\left(\mathrm{mm}^{3}\right)$ & $10700(4600-28000)$ \\
Absolute neutrophil count $\left(\mathrm{mm}^{3}\right)$ & $6400(2000-26000)$ \\
Absolute lymphocyte count $\left(\mathrm{mm}^{3}\right)$ & $2700(1100-10700)$ \\
Hemoglobin $(\mathrm{gr} / \mathrm{dl})$ & $12.6(8.4-16.7)$ \\
Platelets $\left(\mathrm{mm}^{3}\right)$ & $358000(166000-922000)$ \\
C-reactive protein $(\mathrm{mg} / \mathrm{L})$ & $9.6(0.1-158)$ \\
Erythrocyte sedimentation & \\
rate $(\mathrm{mm} / \mathrm{hr})$ & $22(5-118)$ \\
\hline
\end{tabular}

Min.: Minimum; Max. Maximum.

non-nephrotic proteinuria and microscopic hematuria (26.7\%). Only $10.2 \%$ of the whole cohort was referred to pediatric nephrology, mostly due to persistent proteinuria. None of the patients developed acute/chronic kidney failure or end-stage renal disease during the follow-up.

The results of complete blood count and acute phase reactants at the time of diagnosis are given in Table 2 . Leukocytosis with neutrophilia [white blood cell count (WBC): $10700 / \mathrm{mm}^{3}$ (4600-28000), absolute neutrophil count: $\left.6400 / \mathrm{mm}^{3}(2000-26000)\right]$ and mild elevation of acute phase reactants [C-reactive protein (CRP): $9.6 \mathrm{mg} / \mathrm{L}(0.1-158)$, erythrocyte sedimentation rate (ESR): $22 \mathrm{~mm} / \mathrm{hr}(5-118)]$ were observed at the time of diagnosis of HSP.

Demographic, clinical and laboratory features were compared for GI and renal involvement. The results are shown in Table 3. Older age at diagnosis, having lethargy, myalgia, fever, leukocytosis with neutrophilia, higher CRP and ESR values were statistically more common in patients with GI involvement. On the other hand, patients with renal involvement had an older age at diagnosis, higher rates of necrotic rash, subcutaneous edema, abdominal pain, lethargy, myalgia, arthralgia and arthritis. Multiple linear logistic regression analysis showed that none of the above-mentioned parameters was an independent risk factor in predicting the development of GI or renal involvement.

\section{DISCUSSION}

This study has shown that winter and spring are the most common seasons in HSP. The rash is the most common initial manifestation of the disease, but $22.9 \%$ 
TABLE 3. The summary of variables that showed statistical significance regarding gastrointestinal and renal involvement

\begin{tabular}{|c|c|c|c|c|c|c|c|}
\hline \multicolumn{4}{|c|}{ Gastrointestinal involvement } & \multicolumn{4}{|c|}{ Renal involvement } \\
\hline Parameter & Present & Absent & $\mathrm{p}$ & Parameter & Present & Absent & $\mathrm{p}$ \\
\hline Age (years) & 7.9 & 7.3 & 0.044 & Age (years) & 8.8 & 7.4 & 0.002 \\
\hline Lethargy \% & 18.6 & 0.6 & 0.001 & Lethargy \% & 19.7 & 7.2 & 0.003 \\
\hline Myalgia \% & 23.2 & 11.2 & 0.003 & Myalgia \% & 28.9 & 14.1 & 0.004 \\
\hline Fever \% & 7.5 & 2.6 & 0.040 & Necrotic rash \% & 11.8 & 2.8 & 0.004 \\
\hline WBC $\left(\mathrm{mm}^{3}\right)$ & 11600 & 10000 & 0.001 & Subcutaneous edema \% & 73.6 & 59.9 & 0.010 \\
\hline ANC $\left(\mathrm{mm}^{3}\right)$ & 7400 & 6000 & 0.001 & Abdominal pain $\%$ & 61.8 & 50.6 & 0.001 \\
\hline CRP (mg/L) & 11.7 & 7.6 & 0.004 & Arthralgia/arthritis \% & 69.7 & 53.8 & 0.040 \\
\hline $\mathrm{ESR}(\mathrm{mm} / \mathrm{hr})$ & 24 & 21 & 0.002 & & & & \\
\hline
\end{tabular}

Values for continuous variables are given as median. WBC: White blood cell count; ANC: Absolute neutrophil count; CRP: C-reactive protein; ESR: Erythrocyte sedimentation rate.

of patients with HSP do not have a rash as the first manifestation. Abdominal pain, subcutaneous edema and arthralgia are other common initial signs and symptoms in HSP. Renal involvement is seen in a quarter of the patients, but persistent renal involvement is rare. Older age, lethargy and myalgia are common risk factors for $\mathrm{GI}$ and renal involvement.

HSP is the most common form of systemic vasculitis in children. The annual incidence of HSP is $20-86$ per 100000 children $[1,6]$. The disease is most commonly observed during childhood between the ages of 3-15 years and $90 \%$ of the cases occur under the age of 10 years $[1,3]$. The mean age is found to be 7.5 years in one study and 7.9 years in another study $[7,8]$. It affects both genders equally, and in some studies, there is a slightly male predominance. Male/female ratio was 1.08 in the study of Demircioglu Kilic et al. [9] and this number was 1.36 in the cohort of Cakici et al. [10]. Age and gender distribution in our study were compatible with the literature and the median age at diagnosis was 7.5 years and the male/female ratio was 1.15 .

The etiopathogenesis of HSP remains largely unknown. The researchers have focused on abnormal IgA1 glycosylation. It is thought that if a genetically susceptible host, i.e. having galactose deficient variants of $\operatorname{IgA} 1$, is exposed to a trigger, i.e. an infectious agent, the disease manifestations occur $[1,3]$. The seasonal distribution of the disease and history of a preceding infection in some of the cases support this opinion. Wang et al. [8] analyzed seasonal distribution pattern in 16940 patients with HSP and they showed that spring $(31.0 \%)$ was the most common season, and summer (19.0\%) had the smallest number of cases. They analyzed the seasonal distribution of the cases according to age groups and demonstrated that adolescents were more commonly diagnosed during summer $(24.8 \%)$. They also looked for seasonal patterns of eight respiratory and four enteric viruses and concluded that seasonal tendency of HSP was age-dependent and, to some extent, infectious agents, especially influenza and rotavirus, may play an important role in the pathogenesis of the disease. In the study of Anil et al. [11], autumn $(36.0 \%)$ was the most common season and $32.2 \%$ of the patients had a history of recent upper respiratory tract infection (URTI). In the study of Karadag et al. [7], spring $(31.7 \%)$ and winter $(30.2 \%)$ were the two most common seasons. In the same study, $25.2 \%$ of the patients had a history of URTI and $11.6 \%$ had acute gastroenteritis. The results of our study were consistent with the literature that spring (31.2\%) and winter (31.2\%) were the most common seasons, and $29.7 \%$ of the cases had a history of a recent infection. We also think that infectious agents may plan an important role in the etiopathogenesis of HSP.

Musculoskeletal involvement in the form of arthritis, arthralgia are common manifestations during the acute stage of the disease and seen in $50-80 \%$ of the cases. Knees and ankles are the most commonly involved joints, and some patients refuse to walk due to severe arthralgia and/or arthritis. These manifestations are transient and resolve within a few days or weeks $[1,3]$. Trapani et al. [12] reported that the frequency of arthritis and/ 
or arthralgia was 74\% in HSP. Rhim et al. [13] reported that joint involvement was observed in $38.0 \%$ of the patients. Typical purpuric rash is mandatory criteria in childhood HSP classification set [5]. However, some patients with HSP do not present with a typical rash but with joint symptoms. In the study of Trapani et al. [12], $15.0 \%$ of the patients presented with joint manifestations. In our study, arthralgia (57.6\%) and arthritis $(27.6 \%)$ were common at the disease onset and all resolved in less than a week. Joint manifestations were the first manifestation of the disease in $11.8 \%$ of the cases in our cohort. We recommend that if a child presents with acute onset painful joint swelling, especially if accompanied by subcutaneous edema on the dorsum of the hands or feet, the child should be followed closely and the family should be warned for the development of HSP rash.

Gastrointestinal involvement is one of the most important causes of morbidity during the initial phases of the disease. The spectrum of the GI involvement may range from mild abdominal pain to life-threatening intussusception and massive GI bleeding $[1,3,7]$. GI involvement is observed in three-quarters of the children and abdominal pain secondary to bowel angina is the most common form. GI bleeding is seen in $10-20 \%$ of HSP cases $[1,14]$. In one study, abdominal pain was observed in $51.3 \%$ and GI bleeding in $20.5 \%$ of the cases [7]. In another study $56.0 \%$ of the 254 patients with HSP had GI involvement and eight cases (3.1\%) had intussusception [15]. As in joint involvement, GI involvement may be the first sign of the disease in $10-12 \%$ of the cases $[11,12]$. Abdominal pain was observed in $53.3 \%$ of the cases and GI bleeding in $8.0 \%$ of the cases in our study. Moreover, GI involvement was the presenting feature of the disease in $11.1 \%$ of the cases. We also searched for risk factors for GI involvement and found that older age at diagnosis, having lethargy, myalgia, fever, leukocytosis with neutrophilia, higher CRP and ESR values were statistically more common in patients with GI involvement, reflecting more systemic involvement in patients with GI involvement. However, none of the parameters was an independent risk factor for GI involvement.

Renal involvement is the major cause of morbidity and mortality in the long term. The prognosis of a patient with HSP depends on the presence and severity of renal involvement. Renal involvement is seen around $20-60 \%$ of the patients $[1,3,7,8,15-19]$. The most common manifestation of renal involvement is seen as microscopic hematuria and/or non-nephrotic proteinuria that regress spontaneously in most of the cases. Pre- sentation with the nephrotic or nephritic syndrome at disease onset is rare. The review done by Narchi revealed that permanent renal impairment occurred in $1.6 \%$ of those with isolated hematuria and/or non-nephrotic proteinuria and in $19.5 \%$ of the patients who developed a nephritic or nephrotic syndrome. Also, the author stated that $97 \%$ of the cases with renal involvement developed in the first six months of the disease. The recommendation of Narchi was that there was no need to follow up after the first six months in patients with normal urinalysis [18]. The same analysis was carried out by Wang et al. [16] and they found that $91 \%$ of the cases with renal involvement developed in the first six months of the disease. They also recommended six months follow up in patients with normal urinalysis during onset and followups. Overall, the prognosis is good even in severe HSP nephritis. Soylemezoglu et al. [20] reported the course of 443 children with HSP nephritis and they concluded that end-stage renal disease was seen in $1.1 \%$ of the patients. Altugan et al. [17] reported sustained remission by treatment in 18 cases of severe HSP nephritis defined as heavy proteinuria and/or decreased renal function. Renal involvement was seen in $23.5 \%$ of the cases in our cohort. Most of them had transient microscopic hematuria and/or non-nephrotic range proteinuria. None of the patients developed impaired renal function during the follow-up period.

Since renal involvement is the main determinant of prognosis in HSP, many studies have focused on risk factors for the development of renal involvement $[1,3$, $7,8,16,21,22]$. However, it was seen that the same risk factors were not repeatedly found in the studies. Karadag et al. [7] reported that intussusception, relapse and GI involvement were highly associated with renal involvement. Wang et al. [8] searched for risk factors for renal involvement in 2731 children with HSP and stated that age over six years, colder season, late diagnosis (more than eight days between the symptom onset and diagnosis), living in a rural area, recurrence, angioedema, and central nervous system involvement were the significant risk factors for renal involvement. Demircioglu Kilic et al. [9] found that the risk of renal involvement was significantly higher in girls, in patients over 10 years, and in patients with elevated CRP. The meta-analysis of Chan et al. [21] looked for risk factors for renal involvement in 13 studies (2398 children), and they concluded that male gender, diagnosis $>10$ years old, GI involvement, arthritis/arthralgia, persistent rash, relapse, elevated antistreptolysin $\mathrm{O}$, low $\mathrm{C} 3, \mathrm{WBC}>15.000 / \mathrm{mm}^{3}$, and 
thrombocytes $>500.000 / \mathrm{mm}^{3}$ were associated with renal involvement. In our study, we have also searched for risk factors for renal involvement and found that older age at diagnosis, necrotic rash, subcutaneous edema, abdominal pain, lethargy, myalgia, arthralgia and arthritis were significantly higher in patients with renal involvement. However, regression analysis showed that none of the above parameters were independent risk factors for renal involvement.

The major limitation of our study is that this study was retrospectively designed and the duration of followup was short. The strength of our study comes from the dynamics of the cities. Both authors were the first and only pediatric rheumatologists in two big cities with a high population of children, as reflected by the high number of new HSP cases diagnosed only in 22 months. All HSP patients were referred to us from pediatricians, dermatologists, and pediatric nephrologists. Thus, we do not have selection bias, and we believe that our results encompass all the clinical spectrum of HSP patients from the mildest patients to the most severe patients.

\section{Conclusion}

A quarter of children with HSP do not present with a typical rash but abdominal pain or arthritis. If a patient has subcutaneous edema anywhere on the body and abdominal pain or arthritis, the chance of developing an HSP rash in the next few days is high. Our study showed the importance of documentation of constitutional symptoms like fever, myalgia and lethargy at the time of diagnosis. They were associated with a higher risk of GI and renal involvement reflecting more systemic involvement. Although GI and renal involvement were significantly higher in some patients, there was not an independent risk factor to predict renal or GI involvement at the time of diagnosis.

Ethics Committee Approval: This study was approved by the Harran University Clinical Research Ethics Committee (date: 04.11.2019, number: 19/04/01) and was conducted according to the tenets of the declaration of Helsinki.

Conflict of Interest: No conflict of interest was declared by the authors.

Financial Disclosure: The authors declared that this study has received no financial support.

Authorship Contributions: Concept - KO, MC; Design - KO, MC; Supervision - KO, MC; Fundings - KO, MC; Materials - KO, MC; Data collection and/or processing - KO, MC; Analysis and/or interpretation - KO, MC; Literature review - KO; Writing - KO, MC; Critical review $-\mathrm{KO}, \mathrm{MC}$.

\section{REFERENCES}

1. Brogan PA, Bagga A. Leukocytoclastic vasculitis: Henoch-Schönlein purpura and hypersensitivity vasculitis. In: Petty RE, Laxer RM, Lindsey CB, Wedderburn LR, editors. Textbook of Pediatric Rheumatology. $7^{\text {th }}$ ed. Philadelphia: Elsevier; 2016. p. 452-61. [CrossRef]

2. Ozen S, Marks SD, Brogan P, Groot N, de Graeff N, Avcin T, et al. European consensus-based recommendations for diagnosis and treatment of immunoglobulin A vasculitis-the SHARE initiative. Rheumatology (Oxford) 2019;58:1607-16. [CrossRef]

3. Oni L, Sampath S. Childhood IgA Vasculitis (Henoch Schonlein Purpura)-Advances and Knowledge Gaps. Front Pediatr 2019;7:257.

4. Weiss PF. Pediatric vasculitis. Pediatr Clin North Am 2012;59:40723. [CrossRef]

5. Ozen S, Pistorio A, Iusan SM, Bakkaloglu A, Herlin T, Brik R, et al; Paediatric Rheumatology International Trials Organisation (PRINTO). EULAR/PRINTO/PRES criteria for Henoch-Schönlein purpura, childhood polyarteritis nodosa, childhood Wegener granulomatosis and childhood Takayasu arteritis: Ankara 2008. Part II: Final classification criteria. Ann Rheum Dis 2010;69:798-806.

6. Gardner-Medwin JM, Dolezalova P, Cummins C, Southwood TR. Incidence of Henoch-Schönlein purpura, Kawasaki disease, and rare vasculitides in children of different ethnic origins. Lancet 2002;360:1197-202.

7. Karadağ ŞG, Tanatar A, Sönmez HE, Çakmak F, Kiyak A, Yavuz S, et al. The clinical spectrum of Henoch-Schönlein purpura in children: a single-center study. Clin Rheumatol 2019;38:1707-14. [CrossRef]

8. Wang K, Sun X, Cao Y, Dai L, Sun F, Yu P, et al. Risk factors for renal involvement and severe kidney disease in 2731 Chinese children with Henoch-Schönlein purpura: A retrospective study. Medicine (Baltimore) 2018;97:e12520. [CrossRef]

9. Demircioğlu Kiliç B, Kasap Demir B. Determination of Risk Factors in Children Diagnosed With Henoch-Schönlein Purpura. Arch Rheumatol 2018;33:395-401. [CrossRef]

10. Cakici EK, Şükür EDK, Özlü SG, Yazılıtaş F, Özdel S, Gür G, et al. MEFV gene mutations in children with Henoch-Schönlein purpura and their correlations-do mutations matter? Clin Rheumatol 2019;38:1947-52. [CrossRef]

11. Anil M, Aksu N, Kara OD, Bal A, Anil AB, Yavaşcan O, et al. HenochSchönlein purpura in children from western Turkey: a retrospective analysis of 430 cases. Turk J Pediatr 2009;51:429-36.

12. Trapani S, Micheli A, Grisolia F, Resti M, Chiappini E, Falcini F, De Martino M. Henoch Schonlein purpura in childhood: epidemiological and clinical analysis of 150 cases over a 5-year period and review of literature. Semin Arthritis Rheum 2005;35:143-53. [CrossRef]

13. Rhim JW, Lee YT, Kang HM, Suh JS, Lee KY. Changes in clinical features in Henoch-Schönlein purpura during three decades: an observational study at a single hospital in Korea. Clin Rheumatol 2019;38:2811-8. [CrossRef]

14. Ozen S, Bakkaloglu A, Dusunsel R, Soylemezoglu O, Ozaltin F, Poyrazoglu $\mathrm{H}$, et al; Turkish Pediatric Vasculitis Study Group. Childhood vasculitides in Turkey: a nationwide survey. Clin Rheumatol 2007;26:196-200. [CrossRef]

15. Peru H, Soylemezoglu O, Bakkaloglu SA, Elmas S, Bozkaya D, Elmaci AM, et al. Henoch Schonlein purpura in childhood: clinical analysis of 254 cases over a 3-year period. Clin Rheumatol 2008;27:1087-92.

16. Wang H, Das L, Hoh SF, Gao X, Book YX, Arkachaisri T. Urinalysis monitoring in children with Henoch-Schönlein purpura: Is it time to revise? Int J Rheum Dis 2019;22:1271-7. [CrossRef]

17. Altugan FS, Ozen S, Aktay-Ayaz N, Güçer S, Topaloğlu R, Düzova A, 
et al. Treatment of severe Henoch-Schönlein nephritis: justifying more immunosuppression. Turk J Pediatr 2009;51:551-5.

18. Narchi H. Risk of long term renal impairment and duration of follow up recommended for Henoch-Schonlein purpura with normal or minimal urinary findings: a systematic review. Arch Dis Child 2005;90:916-20.

19. Jelusic M, Sestan M, Cimaz R, Ozen S. Different histological classifications for Henoch-Schönlein purpura nephritis: which one should be used?. Pediatr Rheumatol Online J 2019;17:10. [CrossRef]

20. Soylemezoglu O, Ozkaya O, Ozen S, Bakkaloglu A, Dusunsel R, Peru
H, et al; Turkish Pediatric Vasculitis Study Group. Henoch-Schönlein nephritis: a nationwide study. Nephron Clin Pract 2009;112:c199204. [CrossRef]

21. Chan H, Tang YL, Lv XH, Zhang GF, Wang M, Yang HP, Li Q. Risk Factors Associated with Renal Involvement in Childhood HenochSchönlein Purpura: A Meta-Analysis. PLoS One 2016;11:e0167346.

22. Sozeri B, Mir S, Ertan P, Kara OD, Sen S. Rapidly progressive glomerulonephritis in a child with Henoch-Schönlein Vasculitis and familial Mediterranean fever. Pediatr Rheumatol Online J 2009;7:8. [CrossRef] 